\title{
Safety of nalmefene for the treatment of alcohol use disorder: an update
}

\begin{abstract}
Introduction

Reduced drinking has been debated as a treatment goal for heavy drinking alcohol dependent patients, in whom treatment based on abstinence is not always an option. Nalmefene was the first drug approved by the European Medicines Agency (2013) with the indication of reduced drinking in high drinking risk level alcohol dependent patients. Six years after its introduction in Europe, data from clinical experience can be compared with those from preclinical studies and pivotal registration studies to evaluate what nalmefene has added to the treatment of AUD.
\end{abstract}

Areas covered: Systematic review of efficacy and safety data of nalmefene use in humans from preclinical, phase III and phase IV studies, including systematic reviews, meta-analyses, costeffectiveness analyses and other secondary analyses.

Expert opinion: Nalmefene introduces a paradigm change in the treatment of AUD that make it appealing to patients that are reluctant to embrace abstinence, and facilitate patientcentered care in heavy users. However, information regarding safety data in special populations (e.g., patients with alcohol-related diseases, pregnancy, psychiatric disease), and direct comparisons with other potential drugs for alcohol reduction are further needed. Despite the promising role of nalmefene, there are still some factors that limit its wide prescription further than in specialized settings.

Keywords: alcohol use disorders, nalmefene, safety, harm-reduction, adverse effects

\section{Article highlights}

- Three quarters of nalmefene users experienced adverse events in phase III studies whereas $2 / 3$ of the placebo group also reported AEs.

- The most common adverse events were mild/moderate (nausea, dizziness, headache, insomnia) and in most cases did not lead to nalmefene withdrawal (phase III).

- The frequency of AEs in phase IV studies is lower than in phase III RCTs. However, the report of AEs in these studies varies considerably (20-68\%).

- Efficacy results from phase III studies have been confirmed by phase IV studies.

- Phase IV studies provide limited data regarding safety in patients affected by mental health problems.

- Safety data in pregnancy, breastfeeding, severe hepatic impairment and renal impairment are lacking.

\section{Do not disseminate or use without explicit permission from the authors}


Hugo López-Pelayo ${ }^{1}{ }^{*}$, Paola Zuluaga ${ }^{2}$, Elsa Caballeria ${ }^{1}$, Win Van der Brink ${ }^{3}$, Karl Mann $^{4}$, Antoni Gual ${ }^{1 \mathrm{a}}$.

\section{Introduction}

Alcohol related costs are very high in western societies, and exceed by far the revenues obtained from taxation [1]. In Europe, the costs of alcohol use are up to $€ 1,500$ per capita per year [2], with most of the costs associated to binge drinking [3]. Alcohol is linked to more than 200 health conditions [4], in a dose-response manner [5-7]. Alcohol use disorder (AUD) highly contributes to those costs and in fact has been identified as a potentially life-threatening disease. Treatment of AUD remains a challenge, an unmet medical need, for clinicians, researchers and policy makers [8]. Abstinence oriented programs are far from appealing to half of the people with an AUD, and reduced/controlled drinking is well accepted as a treatment goal in primary health settings, but scarcely used with heavy drinkers in specialized care. Several medications (e.g., naltrexone) have shown mixed or negative results in terms of abstinence, but are promising in achieving reductions in drinking $[9,10]$. The potential role of nalmefene for reducing health consequences of alcohol use through reduced drinking is encouraging since evidence shows that reductions on heavy alcohol use are associated with decreased morbidity $[5-7,11]$ and mortality $[7,12]$. Nalmefene is the only medication that has been specifically studied aiming at a reduction goal[13-15]. In addition, modeling studies have shown that nalmefene can be a cost-effective treatment preventing up to 7.179 alcoholattributable diseases and injuries and 309 deaths per 100000 patients compared to psychosocial support alone in a period of 5 years [16-18]. Reduction in 9-year mortality risk due to nalmefene was estimated to be up to $8 \%$ [12]. According to phase III studies, safety and tolerability of nalmefene are good [17]. Since its market-launch, several phase IV studies and meta-analyses have been conducted, which allow an update on safety and tolerability based on real-world samples. Data regarding nalmefene effectiveness will also be revised.

\subsection{Background to the development and use of nalmefene}

In a clinical laboratory setting, nalmefene $(40 \mathrm{mg})$ and naltrexone $(50 \mathrm{mg})$ were tested in social drinkers $(n=90)$ and alcohol dependent patients who were not seeking reduction $(n=125)$ for eight days without a comparison group. Both drugs led to reductions in alcohol use and craving $[19,20]$.

The efficacy of nalmefene use in reducing alcohol consumption was studied in three 24-weeks randomized controlled trials (RCT) (ESENSE I [13] and ESENSE II [14] in Europe, and one study in Japan [21]) and a 52-week RCT (SENSE) [15]. In these trials, selection criteria were $\geq 18$ years old (20 years old in Japan) with a diagnosis of alcohol dependence (DSM-IV-TR), at least 6 heavy drinking days (HDD) ( $\geq 60 \mathrm{~g}$ for men and $\geq 40 \mathrm{~g}$ for women), an average alcohol consumption of at least medium drinking risk level (DRL) according to WHO classification [22], and less than 14 consecutive abstinent days in the four weeks preceding the screening.

In ESENSE I, the effect of as needed nalmefene $20 \mathrm{mg}(\mathrm{n}=306)$ was statistically significant compared to placebo $(n=298)$ in both primary outcomes (number of heavy drinking daysHDDs- and Total Alcohol Consumption -TAC-) and secondary outcomes ( $\gamma$-glutamyltransferase: GGT; alanine aminotransferase: ALAT; aspartate aminotransferase: ASAT; Clinical Global

\section{Do not disseminate or use without explicit permission from} the authors 
Hugo López-Pelayo ${ }^{1}{ }^{*}$, Paola Zuluaga ${ }^{2}$, Elsa Caballeria ${ }^{1}$, Win Van der Brink ${ }^{3}$, Karl Mann $^{4}$, Antoni Gual ${ }^{1 \mathrm{a}}$.

Impression- Severity of IIIness Scale: CGI-S and Global Improvement Scale: CGI-I). ESENSE II also found nalmefene $20 \mathrm{mg}(\mathrm{n}=358)$ to be significantly superior to placebo $(n=360)$ in HDD but not in TAC. Secondary outcomes (ALAT, CGI-S) also improved in favor of nalmefene except for the CGI-I and GGT. The Japanese RCT showed the efficacy of nalmefene $20 \mathrm{mg}(\mathrm{n}=248)$ and nalmefene $10 \mathrm{mg}(n=184)$ versus placebo $(n=245)$ in terms of HDD, TAC, and secondary outcomes (downward shift in DRL of two categories or more, proportion of patients with low or lower DRL, $70 \%$ decrease in TAC, proportion of patients with $\leq 4 \mathrm{HDD}$ ).

A subgroup analyses of ESENSE I plus ESENSE II [23], of patients who had not reduced their alcohol consumption after the first contact with the researchers $(n=667)$ showed at month six improvements in both primary outcomes, HDD (-3.2 95\% Cl -4.8 to $-1.6 ; p<0.00001)$ and TAC ($14.3 \mathrm{~g} /$ day $95 \% \mathrm{Cl}-20.8$ to $-7.8 ; \mathrm{p}=0.00001$ ) as well as in secondary outcomes (GGT and ALAT). A post hoc analyses of ESENSE I plus II aiming to analyze the impact on quality of life [24] $(n=667)$ found that nalmefene was superior to placebo in improving SF-36 mental component (mean difference [MD] $3.0995 \% \mathrm{Cl} 1.29$ to 4.89; $p=0.0008$ ), physical component (MD 1.23 95\% $\mathrm{Cl} 0.15$ to 2.31; $p=0.026$ ), EQ-5D index scores (MD $0.0395 \% \mathrm{Cl} 0.00$ to $0.06 ; p=0.045$ ), EQ-5D health state scores (MD $3.4695 \% \mathrm{Cl} 0.75$ to $6.17 ; \mathrm{p}=0.012$ ), and DrlnC-2R scores (MD -3.22 $95 \% \mathrm{Cl}-6.12$ to $0.33 ; p=0.029)$.

At month 12, in the SENSE study the reduction in the number of HDDs and TAC was statistically significant in favor of nalmefene $(n=310)$ versus placebo $(n=365)$, as well as for GGT and CGI-I, but there were no differences for Carbohydrate deficient transferrin (CDT) or CGI-S.

In all previous studies [13-15,21] both groups received concomitant BRENDA psychosocial intervention [25]. Table 1 shows a summary of efficacy results.

The efficacy of nalmefene in reducing alcohol consumption has also been reviewed in at least four meta-analyses $[10,26-28]$ including both published [13-15,29-31] and unpublished (CPH101-0399, CPH-101-0701, CPH-101-0801) trials. According to Mann et al (2016) [26], random effects were in favor of nalmefene $20 \mathrm{mg}$ for the number of HDDs (Hedges' $\mathrm{g}-0.2095 \% \mathrm{Cl}$ 0.30 to -0.09 ) and for TAC (Hedges' g $-0.3395 \% \mathrm{Cl}-0.48$ to -0.18 ). Higher doses (40mg) did not increase the favorable effects. For the patients with High DRL according to WHO criteria who were not early reducers, the random effects were higher: HDD was $-0.33(95 \% \mathrm{Cl}-0.48$ to -0.18$)$ and for TAC was -0.35 ( $95 \% \mathrm{Cl}-0.51$ to -0.20$)$.

Similarly, a meta-analysis of five RCTs found differences in favor of nalmefene for HDD at month 6 (MD $-1.65,95 \% \mathrm{Cl}-2.41$ to -0.8 ) and at month 12 (MD $-1.60,95 \% \mathrm{Cl}-2.85 ;-0.35$ ), and also for TAC at month 12 (MD $-0.20,95 \% \mathrm{Cl}-0.30$ to -0.10$)$. No benefit was found regarding mortality or quality of life [28].

The comparative effectiveness of drugs used to achieve controlled drinking was explored in two meta-analyses. A network meta-analysis [10] found that nalmefene, baclofen and topiramate were superior to placebo, and that topiramate was superior to other medications (indirect comparison). Nalmefene showed superiority over placebo for TAC (MD -0.19 Cl95\% -

\section{Do not disseminate or use without explicit permission from} the authors 
Hugo López-Pelayo ${ }^{1}$, Paola Zuluaga ${ }^{2}$, Elsa Caballeria ${ }^{1}$, Win Van der Brink ${ }^{3}$, Karl Mann ${ }^{4}$, Antoni Gual $^{1 \mathrm{a}}$.

0.29 to -0.10 ) and for HDD (MD $-0.2295 \% \mathrm{Cl}-0.32$ to -0.12 ). This meta-analysis did not include studies with enough power for exploring health outcomes.

Finally, an indirect meta-analysis [27] of 17 RCTs, including 4 RCTs of nalmefene and 13 RCTs of naltrexone did not find statistical significance differences between the two active medications.

\section{Body of review}

\subsection{Mechanism of action}

As a $\mu$ and $\delta$-opioid antagonist, nalmefene reduces the pleasant and positive reinforcing properties of alcohol[32,33], and as a k-opioid partial agonist it probably enhances its sedative and dysphoric properties and also reduces craving for alcohol and impulsivity that promotes alcohol drinking [32].

Nalmefene further differs from naltrexone by its longer plasma half-life, higher bioavailability and lower liver toxicity [29,32], and more effective binding to central opioid receptors as naltrexone blocks on $\delta$-opioid receptors to a smaller extent than nalmefene [34]. It is rapidly absorbed after oral administration, peak plasma level is reached within $90 \mathrm{~min}$ after ingestion and half-life is $13.4 \mathrm{~h}$ after a single dose. Nalmefene occupies more than $80 \%$ of the MOR receptors up to 72 hours $[35,36]$. Nalmefene is extensively metabolized in the liver, largely by glucuronidation rather than turn into a different metabolite.

Unlike naltrexone, nalmefene is also a partial agonist for K-opioid receptor (KOR) in humans [37]. Chronic alcohol exposition enhances the KOR system activity in the nucleus accumbens that leads to a negative emotional state when alcohol consumption is decreased or stopped, which is followed by craving and negative reinforcement when alcohol again becomes available. Preclinical data indicate that the modulation of KOR system decreases dependenceinduced alcohol self-administration [38].

As with naltrexone, there are no clinical safety concerns around its co-ingestion with alcohol[39], and in vitro studies have indicated that it has not relevant interactions with other drugs metabolized by the CYP450 and uridine 50-diphospho-glucuronosyltransferase (UDP)glucuronosyltransferase (UGT) systems. Chronic use of potent inhibitors of the UGT2B7 system (e.g. diclofenac) may increase nalmefene levels, but occasional use does not [40].

\subsection{Clinical applications}

\section{Phase IV studies}

Four studies have been conducted in specialized treatment units $(n=110)[41,42]$, primary care settings $(n=330)$ [43] and in psychiatric outpatients $(n=65, n=25)[44,45]$. They were single$\mathrm{arm} /$ naturalistic $[41,42,44]$ or open-label studies [43] with follow-ups ranging from eight weeks to six months. In these studies it was found that nalmefene decreased HDD and TAC, and improved secondary outcomes, including reduction in daily alcohol consumption of at least $70 \%$, a downshift of two categories in the DRL (WHO), a shift to the LR category, Borderline

\section{Do not disseminate or use without explicit permission from} the authors 
Hugo López-Pelayo ${ }^{1}$, Paola Zuluaga ${ }^{2}$, Elsa Caballeria ${ }^{1}$, Win Van der Brink ${ }^{3}$, Karl Mann ${ }^{4}$, Antoni Gual $^{1 \mathrm{a}}$.

Symptom List-23 and the Clinical Global Impression Scale for Borderline Personality Disorder. For more details see table 1.

\subsection{Safety evaluation}

\subsubsection{Safety in pre-marketing studies}

Within the safety and tolerability analysis of randomized phase III studies of nalmefene versus placebo -ESENSE1 [13], ESENSE2 [14] and SENSE [23]-, up to 75\% of patients in the nalmefene group and $63 \%$ in the placebo group reported adverse events (AEs). These differences were statistically significant in the ESENSE1 and SENSE studies. Nausea, dizziness, headache, and insomnia were the most frequent symptoms in the nalmefene group, with $13 \%$ of the patients abandoning the medication, compared to $6 \%$ in the placebo group. Most AEs were transient, with an average length of 3 to 7 days and occurred during the first days of medication use. Psychiatric AEs were reported in $2.9 \%$ of the patients, mainly confusional symptoms, generally occurring after the first dose and with a short duration [17].

The frequency of AEs in the Japanese study was $86.6 \%$ in the nalmefene group compared to $79.2 \%$ in the placebo group [21]. AEs were similar to those in the previously mentioned trials, being most mild or moderate in severity, and leading to discontinuation in about $2 \%$ of patients for either the $20 \mathrm{mg}$ or $10 \mathrm{mg}$ nalmefene. The $10 \mathrm{mg}$ dose was associated with about $5 \%$ lower rates of dizziness, malaise, somnolence and vomiting compared to the $20 \mathrm{mg}$ dose. The rates of other AEs were generally similar between the two doses.

In the Japanese study, serious $\mathrm{AE}(\mathrm{SAE})$ were reported in all treatment groups, occurring in two patients $(0.8 \%)$ in the nalmefene $20 \mathrm{mg}$ group, two patients $(1.1 \%)$ in nalmefene $10 \mathrm{mg}$ group, and two patients $(0.8 \%)$ in the placebo group. SAEs in the nalmefene group were chronic hepatitis and gastroenteritis at a dose of $20 \mathrm{mg}$, femur fracture and spinal compression fracture (both occurred in the same patient), and one death at a dose of $10 \mathrm{mg}$. The cause of death was unknown and its association with the treatment could not be ruled out.

\subsubsection{Post-marketing data}

In the study conducted by Barrio et al, with 110 patients, 29 patients (26.4\%) presented medication-related adverse events during the first month of treatment. The most frequent AEs were dizziness, nausea, somnolence, and cognitive numbness. No SAEs were recorded [41]. At six months, no new drug-related AEs were notified [42].

Results from the multicenter open-label study by Castera et al (2019) [43], point out that nalmefene was well tolerated. From the 330 patients included, 19 (6.4\%) withdrew from the study due to adverse events (mainly nausea and dizziness). Overall, $68 \%$ of patients in the nalmefene cohort had one or more AEs, most of which were mild or moderate in severity. A total of 22 patients (7\%) reported SAEs, describing alcohol withdrawal syndrome, depressive symptoms and hepatic cirrhosis.

\section{Do not disseminate or use without explicit permission from} the authors 
Hugo López-Pelayo ${ }^{1}$, Paola Zuluaga ${ }^{2}$, Elsa Caballeria ${ }^{1}$, Win Van der Brink ${ }^{3}$, Karl Mann ${ }^{4}$, Antoni Gual $^{1 \mathrm{a}}$.

In the Di Nicola et al study [44] with 65 patients, 24 patients (38.5\%) showed AEs, being dizziness, nausea, headache and insomnia the most common. These AEs mostly occurred after the first dose, had a short duration and were mild or moderate in intensity. Similarly, MartinBlanco et al[45], found that the most common adverse events were dizziness, nausea, decreased appetite, and unsteady gait $(n=5,20 \%)$. Two patients withdraw the medication due to nausea, dizziness, and unsteady gait $(n=1)$ and nausea and malaise $(n=1)$ [45].

A meta-analyses of eight RCTs found no increased risk of SAEs in patients with substance use or impulse control disorder $(n=1,828)$ treated with nalmefene compared to placebo $(n=1,119)$ [46]. However, patients taking nalmefene were 3.22 times more likely to withdraw due to adverse events. In the withdrawal stratified analysis, no statistical differences among patients with substance use or impulse control disorder were observed. The risk of withdrawal due to adverse events of nalmefene was also supported by the meta-analysis of Jonas et al. (2014) [47].

Table 2 shows a summary of adverse events from phase III and IV studies.

\subsubsection{Safety in special populations (Table 3).}

\subsubsection{Opiate-dependent patients.}

Nalmefene, being a modulator of the opioid system, interacts with other opioids (methadone, buprenorphine, codeine) and may precipitate an opiate withdrawal syndrome. It has been reported in patients with opioid abuse (oxycodone or codeine) and in maintenance therapy with methadone and buprenorphine [48]. The development of withdrawal syndrome can occur in a variable range of severity, requiring medical observation in most of the cases and even treatment in intensive care, as reported in two cases [49]. For this reason it is recommended to document the use of other substances and to be cautious in those patients with opioid abuse or under opioid treatment for pain or opioid dependence [50].

\subsubsection{Psychiatric disorder}

Nalmefene has been studied in patients with stable psychiatric diseases $[15,42]$ showing that patients with AUD and psychiatric comorbidity $(n=42)$ experienced adverse events after 6 months of nalmefene use with the same frequency as the general population (40\%) [44]. Also, in a small open non-controlled trial carried out in 25 individuals with Borderline Personality Disorder and AUD, nalmefene showed a good tolerability and only five out of 25 participants (20\%) reported mild AEs, that led to the cessation of the treatment in only two of the cases (8\%) [45]. The development of psychotic symptoms 48 hours after starting nalmefene treatment has been reported in a patient with schizoaffective disorder[51], and two patients

\section{Do not disseminate or use without explicit permission from the authors}


Hugo López-Pelayo ${ }^{1}{ }^{*}$, Paola Zuluaga $^{2}$, Elsa Caballeria ${ }^{1}$, Win Van der Brink ${ }^{3}$, Karl Mann ${ }^{4}$, Antoni Gual ${ }^{1 a}$.

with a history of depressive disorder [52] presented somnolence, asthenia and fatigue 48 hours after nalmefene use [52]. These symptoms remitted after drug discontinuation. One case of severe central sleep apnea after 4 months of treatment with nalmefene was reported in a woman with depressive disorder and obesity [53].

\subsubsection{Renal impairment}

Pharmacokinetic studies pointed out that the volume of distribution of nalmefene was significantly higher and the total body clearance lower in patients with End-Stage Renal Disease compared to patients with normal renal function [54]. No significant impairment of kidney function was reported during the use of nalmefene in alcohol dependent patients [46]. Although the use of opioid system modulators is not recommended in patients with severe renal failure, a direct toxicity on kidney function can be excluded and dose adjustment is not required in mild to moderate renal failure [55].

\subsubsection{Hepatic impairment}

The clearance of nalmefene was found to be significantly reduced in a small study of 12 patients with liver disease compared to control subjects. The reduction in the clearance was inversely proportional to the severity of liver disease and also the changes in glucuronidation may only occur with more advanced disease [56]. Although there is no evidence of hepatotoxicity associated with nalmefene [57], there is a lack of data involving patients with advanced liver disease. Furthermore, abstinence is the preferred treatment goal for this group of patients, which is not the intended treatment goal of nalmefene [58].

\subsubsection{Pregnancy and breastfeeding}

Data on the effects of nalmefene in these two conditions are lacking.

\subsubsection{Pharmacogenomics data}

The only pharmacogenomics analysis of a randomized placebo-controlled multicenter study [31] found no evidence that allelic variation in opioid receptor genes moderated the response to nalmefene treatment [59].

\subsubsection{Comparison with the safety of other drugs}

\section{Do not disseminate or use without explicit permission from the authors}


Hugo López-Pelayo ${ }^{1}$, Paola Zuluaga ${ }^{2}$, Elsa Caballeria ${ }^{1}$, Win Van der Brink ${ }^{3}$, Karl Mann ${ }^{4}$, Antoni Gual $^{1 \mathrm{a}}$.

A cohort study with medical administrative data from the French national health insurance information system database (SNIIRAM) included patients aged 18 to 70 years, with no serious comorbidities initiating baclofen or approved medications for the treatment of alcohol dependence (nalmefene, naltrexone, acamprosate) between 2009 and 2015 [60]. Primary and secondary outcomes at one year follow-up were death from any cause and death due to specific causes, respectively. The study included 165.334 patients, $28.8 \%$ exposed to baclofen and $71.2 \%$ exposed to the other approved medications. After multivariate analysis, the risk of hospitalization and death was higher for patients treated with baclofen versus approved medications (HRf $=1.13[1.09-1.17]$ and $H R f=1.31[1.08-1.60]$, respectively). Despite the increase in hospitalization and mortality in patients treated with baclofen, in a recent systematic review and meta-analysis with 6036 patients and $32 \mathrm{RCT}$, no differences were found for any treatment (nalmefene, naltrexone, topiramate, baclofen, acamprosate) on mortality and serious adverse events. However, more adverse events and withdrawals for safety reasons were evidenced for naltrexone and nalmefene, and withdrawals increased for nalmefene [10].

In a meta-analysis of the benefits and harms of medications (US FDA-approved and others) for adults with AUD, patients treated with naltrexone or nalmefene had a higher risk of withdrawal due to adverse events with a Number Need to Harm (NNH) for naltrexone of 48 ( $95 \% \mathrm{Cl}, 30$ to $112 ; 17$ trials, $\mathrm{n}=2743$ ); and $\mathrm{NNH}$ for nalmefene of $12(95 \% \mathrm{Cl}, 7$ to $50 ; 5$ trials, $\mathrm{n}=2054$ ), compared with placebo. In the analysis for acamprosate or topiramate no significant differences compared to placebo were found. Those treated with nalmefene had a higher risk of dizziness, headache, insomnia nausea and vomiting [47].A randomized study to receive placebo, naltrexone (titrated to $50 \mathrm{mg} /$ day), or nalmefene (titrated to $40 \mathrm{mg} /$ day) among non treatment-seeking alcoholics and social drinkers, patients treated with nalmefene reported significantly higher levels of mild/moderate AEs as poor sleep, irritability, nausea/vomiting and impaired attention in comparison to placebo and naltrexone [19].

\section{Conclusion}

Nalmefene has shown its efficacy in short-term (24-weeks) and long-term studies (52-weeks) in at least four studies under the reduction paradigm, especially in those patients with a higher drinking risk level. These results have been confirmed by meta-analyses and post-marketing studies. Cost-effectiveness is promising according to several simulations but further studies on

\section{Do not disseminate or use without explicit permission from the authors}


Hugo López-Pelayo ${ }^{1}{ }^{*}$, Paola Zuluaga $^{2}$, Elsa Caballeria ${ }^{1}$, Win Van der Brink ${ }^{3}$, Karl Mann ${ }^{4}$, Antoni Gual ${ }^{1 a}$.

this topic are needed. Morbidity and mortality must be taken into account as main outcomes in observational studies and clinical trials.

Most patients (70-90\%) treated with nalmefene during the phase III studies had adverse events but these were mild to moderate allowing in most cases to continue the treatment. Although the frequency of adverse events in post-marketing studies is similar to those observed in clinical studies involving more patients with extensive follow-up are needed. Also, only two-phase IV studies provide data on the frequency of withdrawal due to AEs. Differences in the frequency of AEs between the studies could be attributed both to the differences in their design, the greater number of patients and the closer monitoring of AEs in RCTs.

Safety of nalmefene is similar to other drugs according to observational studies and metaanalyses. The increased reporting of CNS symptoms (dizziness, insomnia, confusion/disorientation) in patients treated with nalmefene in comparison to naltrexone, despite having a similar safety profile, may be due to nalmefene action as a partial agonist at the KOR system. However, it is not known whether this difference in kappa-opioid receptors activity between the two drugs is reflected in differences in their efficacy in reducing drinking or in side effects.

Interpretation of the findings must be prudent due to the fact that most of the existing literature is sponsored by the industry.

\section{Expert opinion}

Although naltrexone is used in several countries with the goal of reducing alcohol consumption in patients with moderate or severe AUD[61], nalmefene was the first and only treatment approved in Europe specifically for reduced drinking in alcohol dependent patients. In comparison to other approaches, this reduction goal may enhance treatment participation of AUD patients who feel not able or are not willing to stop drinking completely. It is the first and only medication for alcohol dependence that is used "as-needed" which may enhance selfefficacy and patient empowerment.

Alcohol reduction goal was available for a long time in mild/moderate AUD patients. With some exceptions (e.g., The Sinclair Method [62]), abstinence used to be the unique goal available for heavy users before nalmefene approval. Nalmefene facilitate patient-centered

\section{Do not disseminate or use without explicit permission from the authors}


Hugo López-Pelayo ${ }^{1}{ }^{*}$, Paola Zuluaga $^{2}$, Elsa Caballeria ${ }^{1}$, Win Van der Brink ${ }^{3}$, Karl Mann ${ }^{4}$, Antoni Gual ${ }^{1 a}$.

care in heavy users [42] according to patients' preferences, values and ongoing context. The offer of treatments with a reduction goal may be appealing to large numbers of patients who are reluctant to embrace an abstinence oriented program.

Nalmefene introduces a paradigm change in the treatment of AUD. On one hand it may be appealing to many physicians, since it avoids the 'abstinence goal' and decreases the risk of labeling and stigmatization. On the other hand, it implies a shift in routine clinical practice that may be difficult, especially in specialized settings.

Besides that, the drug has faced relevant problems in most of the EU countries concerning the co-payment by the Health system, leading to lower prescription rates.

Safety data in populations with alcohol-related diseases (e.g. hepatic or cognitive impairment), and in breastfeeding and pregnancy are still needed. Further information is needed to know the cost-effectiveness of nalmefene, specifically regarding health outcomes and direct/indirect costs. Morbidity and mortality outcomes must be assessed specifically in populations where the reduction approach is controversial, such as alcoholic-liver disease or alcohol-related cognitive impairment.

Direct comparisons with other potential drugs for alcohol reduction are also needed (both for safety and effectiveness reasons). Long-term efficacy has been studied just in one phase III study. There is no data of long-term efficacy beyond 12 months or in phase IV studies.

In our opinion, and according to the results from the studies reviewed in the present work, in 5 years' time, nalmefene should be widely available for its prescription in different settings, especially in primary care and in the emergency room. However, given its evolution in recent years, it is quite likely that the drug will remain mostly available in specialized settings.

The recently published Study from Japan has given new positive data to the potential use of nalmefene. This will be especially true if the drug should be approved in Japan.

\section{Do not disseminate or use without explicit permission from the authors}


Hugo López-Pelayo ${ }^{1}$, Paola Zuluaga ${ }^{2}$, Elsa Caballeria ${ }^{1}$, Win Van der Brink ${ }^{3}$, Karl Mann ${ }^{4}$, Antoni Gual $^{1 \mathrm{a}}$.

[1] Blanchette JG, Chaloupka FJ, Naimi TS. The Composition and Magnitude of Alcohol Taxes in States: Do They Cover Alcohol-Related Costs? J. Stud. Alcohol Drugs [Internet]. 2019 [cited 2019 Sep 17];80:408-414. Available from: https://www.jsad.com/doi/10.15288/jsad.2019.80.408.

[2] Barrio P, Reynolds J, García-Altés A, et al. Social costs of illegal drugs, alcohol and tobacco in the European Union: A systematic review. Drug Alcohol Rev. 2017;36:578588.

[3] Sacks JJ, Gonzales KR, Bouchery EE, et al. 2010 National and State Costs of Excessive Alcohol Consumption. Am. J. Prev. Med. 2015;49:e73-e79.

[4] World Health Organization. Global status report on alcohol and health 2018. World Heal. World Health Organization, editor. Geneva; 2018.

[5] Rehm J, Hasan OSM, Imtiaz S, et al. Quantifying the contribution of alcohol to cardiomyopathy: A systematic review. Alcohol [Internet]. 2017;61:9-15. Available from: http://dx.doi.org/10.1016/j.alcohol.2017.01.011.

[6] Cherpitel CJ, Ye Y, Monteiro MG. Dose-Response Relative Risk of Injury From Acute Alcohol Consumption in 22 Countries: Are Women at Higher Risk Than Men? Alcohol Alcohol. 2019;54:396-401.

[7] Cherpitel CJ, Witbrodt J, Korcha RA, et al. Dose-Response Relationship of Alcohol and Injury Cause: Effects of Country-Level Drinking Pattern and Alcohol Policy. Alcohol. Clin. Exp. Res. 2019;43:850-856.

[8] Han B, Compton WM, Blanco C, et al. Prevalence, treatment, and unmet treatment needs of us adults with mental health and substance use disorders. Health Aff. 2017;36:1739-1747.

[9] Kranzler HR, Feinn R, Morris P, et al. A meta-analysis of the efficacy of gabapentin for treating alcohol use disorder. Addiction. 2019;1547-1555.

[10] Palpacuer C, Duprez R, Huneau A, et al. Pharmacologically controlled drinking in the treatment of alcohol dependence or alcohol use disorders: a systematic review with direct and network meta-analyses on nalmefene, naltrexone, acamprosate, baclofen and topiramate. Addiction. 2018;113:220-237.

[11] Witkiewitz K, Hallgren KA, Kranzler HR, et al. Clinical Validation of Reduced Alcohol Consumption After Treatment for Alcohol Dependence Using the World Health Organization Risk Drinking Levels. Alcohol. Clin. Exp. Res. 2017;41:179-186.

[12] Roerecke M, Gual A, Rehm J. Reduction of alcohol consumption and subsequent mortality in alcohol use disorders: Systematic review and meta-analyses. J. Clin. Psychiatry. 2013;74.

[13] Mann K, Bladström A, Torup L, et al. Extending the treatment options in alcohol dependence: A randomized controlled study of As-needed nalmefene. Biol. Psychiatry. 2013;73:706-713.

[14] Gual A, He Y, Torup L, et al. A randomised, double-blind, placebo-controlled, efficacy

Do not disseminate or use without explicit permission from the authors 
Hugo López-Pelayo ${ }^{1}$, Paola Zuluaga ${ }^{2}$, Elsa Caballeria ${ }^{1}$, Win Van der Brink ${ }^{3}$, Karl Mann ${ }^{4}$, Antoni Gual $^{1 \mathrm{a}}$.

study of nalmefene, as-needed use, in patients with alcohol dependence. Eur. Neuropsychopharmacol. [Internet]. 2013;23:1432-1442. Available from: http://dx.doi.org/10.1016/j.euroneuro.2013.02.006.

[15] van den Brink W, Sørensen P, Torup L, et al. Long-term efficacy, tolerability and safety of nalmefene as-needed in patients with alcohol dependence: A 1-year, randomised controlled study. J. Psychopharmacol. [Internet]. 2014 [cited 2019 Sep 9];28:733-744. Available from: http://journals.sagepub.com/doi/10.1177/0269881114527362.

[16] Laramée P, Brodtkorb TH, Rahhali N, et al. The cost-effectiveness and public health benefit of nalmefene added to psychosocial support for the reduction of alcohol consumption in alcohol-dependent patients with high/very high drinking risk levels: A Markov model. BMJ Open. 2014;4.

[17] Van Den Brink W, Strang J, Gual A, et al. Safety and tolerability of as-needed nalmefene in the treatment of alcohol dependence: Results from the Phase III clinical programme. Expert Opin. Drug Saf. 2015;14:495-504.

**This study offers the pooled data from three randomised, placebo controlled studies on the safety and tolerability of nalmefene $18 \mathrm{mg}$ in patients with alcohol dependance.

[18] Millier A, Laramée P, Rahhali N, et al. Cost-effectiveness of nalmefene added to psychosocial support for the reduction of alcohol consumption in alcohol-dependent patients with high/very high drinking risk levels: A microsimulation model. J. Stud. Alcohol Drugs. 2017;78:867-876.

[19] Drobes DJ, Anton RF, Thomas SE, et al. A clinical laboratory paradigm for evaluating medication effects on alcohol consumption: Naltrexone and nalmefene. Neuropsychopharmacology. 2003;28:755-764.

[20] Drobes DJ, Anton RF, Thomas SE, et al. Effects of naltrexone and nalmefene on subjective response to alcohol among non-treatment-seeking alcoholics and social drinkers. Alcohol. Clin. Exp. Res. 2004;28:1362-1370.

[21] Miyata H, Takahashi M, Murai Y, et al. Nalmefene in alcohol-dependent patients with a high drinking risk: Randomized controlled trial. Psychiatry Clin. Neurosci. 2019

[22] World Health Organization. International Guide for MonitoringAlcohol Consumption and Related Harm. [Internet]. World Heal. Organ. 2000 [cited 2019 Sep 30]. Available from: ttp://apps.who.int/iris/bitstream/10665/66529/1/WHO_MSD_MSB_00.4.pdf.

[23] van den Brink W, Aubin HJ, Bladström A, et al. Efficacy of As-Needed Nalmefene in Alcohol-Dependent Patients with at Least a High Drinking Risk Level: Results from a Subgroup Analysis of Two Randomized Controlled 6-Month Studies. Alcohol Alcohol. 2013;48:570-578.

[24] François C, Rahhali N, Chalem $\mathrm{Y}$, et al. The effects of as-needed nalmefene on patientreported outcomes and quality of life in relation to a reduction in alcohol consumption in alcohol-dependent patients. PLoS One. 2015;10:1-13.

[25] Starosta AN, Leeman RF, Volpicelli JR. The BRENDA model: integrating psychosocial

Do not disseminate or use without explicit permission from the authors 
Hugo López-Pelayo ${ }^{1}$, Paola Zuluaga ${ }^{2}$, Elsa Caballeria ${ }^{1}$, Win Van der Brink ${ }^{3}$, Karl Mann ${ }^{4}$, Antoni Gual $^{1 \mathrm{a}}$.

treatment and pharmacotherapy for the treatment of alcohol use disorders. J. Psychiatr. Pract. [Internet]. 2006 [cited 2019 Sep 9];12:80-89. Available from: http://www.ncbi.nlm.nih.gov/pubmed/16728904.

[26] Mann K, Torup L, Sørensen P, et al. Nalmefene for the management of alcohol dependence: review on its pharmacology, mechanism of action and meta-analysis on its clinical efficacy. Eur. Neuropsychopharmacol. [Internet]. 2016 [cited 2019 Sep 9];26:1941-1949. Available from:

https://linkinghub.elsevier.com/retrieve/pii/S0924977X16319708.

** This paper offers a concise overview on nalmefene neuropharmacological mechanisms, and also sistematically reviews the literature on the efficacy of this drug for the reduction of alcohol use.

[27] Soyka M, Friede M, Schnitker J. Comparing Nalmefene and Naltrexone in Alcohol Dependence: Are there any Differences? Results from an Indirect Meta-Analysis. Pharmacopsychiatry. 2016;49:66-75.

[28] Palpacuer C, Laviolle B, Boussageon R, et al. Risks and Benefits of Nalmefene in the Treatment of Adult Alcohol Dependence: A Systematic Literature Review and MetaAnalysis of Published and Unpublished Double-Blind Randomized Controlled Trials. PLoS Med. 2015;12:1-17.

[29] Mason BJ, Salvato FR, Williams LD, et al. A double-blind, placebo-controlled study of oral nalmefene for alcohol dependence. Arch. Gen. Psychiatry. 1999;56:719-724.

[30] Anton RF, Pettinati H, Zweben A, et al. A multi-site dose ranging study of nalmefene in the treatment of alcohol dependence. J. Clin. Psychopharmacol. 2004;24:421-428.

[31] Karhuvaara S, Simojoki K, Virta A, et al. Targeted nalmefene with simple medical management in the treatment of heavy drinkers: A randomized double-blind placebocontrolled multicenter study. Alcohol. Clin. Exp. Res. 2007;31:1179-1187.

[32] Ingman K, Hagelberg N, Aalto S, et al. Prolonged central $\mu$-opioid receptor occupancy after single and repeated nalmefene dosing. Neuropsychopharmacology. 2005;30:2245-2253.

[33] Bencherif B, Wand GS, McCaul ME, et al. Mu-opioid receptor binding measured by [11C]carfentanil positron emission tomography is related to craving and mood in alcohol dependence. Biol. Psychiatry. 2004;55:255-262.

[34] Shen WW. Anticraving therapy for alcohol use disorder: A clinical review. Neuropsychopharmacol. Reports. 2018;38:105-116.

[35] Gal TJ, DiFazio CA, Dixon R. Prolonged blockade of opioid effect with oral nalmefene. Clin. Pharmacol. Ther. 1986;40:537-542.

[36] Dixon R, Gentile J, Hsu H-B, et al. Nalmefene: Safety and Kinetics After Single and Multiple Oral Doses of a New Opioid Antagonist. J. Clin. Pharmacol. [Internet]. 1987 [cited 2019 Sep 10];27:233-239. Available from: http://doi.wiley.com/10.1002/j.15524604.1987.tb02191.x.

\section{Do not disseminate or use without explicit permission from} the authors 
Hugo López-Pelayo ${ }^{1}{ }^{*}$, Paola Zuluaga $^{2}$, Elsa Caballeria ${ }^{1}$, Win Van der Brink ${ }^{3}$, Karl Mann ${ }^{4}$, Antoni Gual ${ }^{1 a}$.

[37] Bart G, Schluger JH, Borg L, et al. Nalmefene induced elevation in serum prolactin in normal human volunteers: Partial kappa opioid agonist activity?

Neuropsychopharmacology. 2005;30:2254-2262.

[38] Walker BM, Koob GF. Pharmacological evidence for a motivational role of $\mathrm{k}$-opioid systems in ethanol dependence. Neuropsychopharmacology. 2008;33:643-652.

[39] Sinclair JMA, Chambers SE, Shiles CJ, et al. Safety and Tolerability of Pharmacological Treatment of Alcohol Dependence: Comprehensive Review of Evidence. Drug Saf. 2016;39:627-645.

[40] Selincro | European Medicines Agency [Internet]. [cited 2019 Sep 10]. Available from: https://www.ema.europa.eu/en/medicines/human/EPAR/selincro.

[41] Barrio P, Ortega L, Guardia J, et al. Who Receives Nalmefene and How Does It Work in the Real World? A Single-Arm, Phase IV Study of Nalmefene in Alcohol Dependent Outpatients: Baseline and 1-Month Results. Clin. Drug Investig. 2018;38:147-155.

[42] Barrio P, Roncero C, Ortega L, et al. The More You Take It, the Better It Works: SixMonth Results of a Nalmefene Phase-IV Trial. J. Clin. Med. 2019;8:471.

[43] Castera P, Stewart E, Großkopf J, et al. Nalmefene, Given as Needed, in the Routine Treatment of Patients with Alcohol Dependence: An Interventional, Open-Label Study in Primary Care. Eur. Addict. Res. 2019;24:293-303.

*The authors conducted an open-label study in a primary care setting, and present the favourable results regarding the efficacy of nalmefene $18 \mathrm{mg}$ in conjuntion with psychosocial support for the reduction in alcohol consumption. The results regarding tolerability are also presented.

[44] Di Nicola M, De Filippis S, Martinotti G, et al. Nalmefene in Alcohol Use Disorder Subjects with Psychiatric Comorbidity: A Naturalistic Study. Adv. Ther. [Internet]. 2017 [cited 2019 Sep 9];34:1636-1649. Available from: http://link.springer.com/10.1007/s12325-017-0546-3.

*This study presents the efficacy and tolerability results in a naturalistic study conducted in patients with alcohol use disorder and stabilized psychiatric comorbidities. The results found are relevant as suggest that nalmefene would be a therapeutic option in clinical settings where comorbidities are frequent.

[45] Martín-Blanco A, Patrizi B, Soler J, et al. Use of nalmefene in patients with comorbid borderline personality disorder and alcohol use disorder. Int. Clin. Psychopharmacol. [Internet]. 2017;32:231-234. Available from: http://insights.ovid.com/crossref?an=00004850-201707000-00008.

[46] Johansen KGV, Tarp S, Astrup A, et al. Harms associated with taking nalmefene for substance use and impulse control disorders: A systematic review and meta-analysis of randomised controlled trials. DeLuca V, editor. PLoS One [Internet]. 2017;12:e0183821. Available from: https://dx.plos.org/10.1371/journal.pone.0183821.

[47] Jonas DE, Amick HR, Feltner C, et al. Pharmacotherapy for adults with alcohol use disorders in outpatient settings: A systematic review and meta-analysis. JAMA - J. Am.

Do not disseminate or use without explicit permission from the authors 
Hugo López-Pelayo ${ }^{1}$, Paola Zuluaga ${ }^{2}$, Elsa Caballeria ${ }^{1}$, Win Van der Brink ${ }^{3}$, Karl Mann ${ }^{4}$, Antoni Gual $^{1 \mathrm{a}}$.

Med. Assoc. 2014;311:1889-1900.

[48] Dahmke H, Kupferschmidt $H$, Kullak-Ublick GA, et al. Nalmefen und OpioidEntzugssyndrom: systematische Analyse der globalen Pharmacovigilance-Datenbank. Praxis (Bern. 1994). 2015;104:1129-1134.

[49] Donnerstag N, Schneider T, Lüthi A, et al. Severe opioid withdrawal syndrome after a single dose of nalmefene. Eur. J. Clin. Pharmacol. [Internet]. 2015;71:1025-1026. Available from: http://dx.doi.org/10.1007/s00228-015-1884-1.

[50] Franchitto N, Jullian B, Salles J, et al. Management of precipitated opiate withdrawal syndrome induced by nalmefene mistakenly prescribed in opiate-dependent patients: a review for clinicians. Expert Opin. Drug Metab. Toxicol. 2017;13:669-677.

[51] Salles J, Ponté C, Schmitt L. Psychotic Decompensation During Nalmefene Treatment in a Patient With Schizoaffective Disorder: A Case Report. J. Dual Diagn. [Internet]. 2019;15:118-121. Available from: https://doi.org/10.1080/15504263.2019.1574367.

[52] Milano G, Zavan V, Natta WM, et al. Clinical experience about an unexpected adverse event during nalmefene treatment in two patients with alcohol use disorder. J. Clin. Pharm. Ther. 2016;41:97-100.

[53] Khouri C, Arbib F, Revol B, et al. Severe central sleep apnoea associated with nalmefene: a case report. Br. J. Clin. Pharmacol. 2018;84:1075-1076.

[54] Matzke GR, Frye RF, Alexander ACM, et al. The effect of renal insufficiency and hemodialysis on the pharmacokinetics of nalmefene. J. Clin. Pharmacol. 1996;36:144151.

[55] Antonelli M, Ferrulli A, Sestito L, et al. Alcohol addiction - the safety of available approved treatment options. Expert Opin. Drug Saf. [Internet]. 2018;17:169-177. Available from: https://doi.org/10.1080/14740338.2018.1404025.

[56] Frye RF, Matzke GR, Schade R, et al. Effects of liver disease on the disposition of the opioid antagonist nalmefene. Clin. Pharmacol. Ther. 1997;61:15-23.

[57] Leggio L, Lee MR. Treatment of Alcohol Use Disorder in Patients with Alcoholic Liver Disease. Am. J. Med. [Internet]. 2017;130:124-134. Available from: http://dx.doi.org/10.1016/j.amjmed.2016.10.004.

[58] Fuster D, Samet JH. Alcohol use in patients with chronic liver disease. N. Engl. J. Med. 2018;379:1251-1261.

[59] Arias A, Armeli S, Gelernter J. Effects of Opioid Receptor Gene Variation. 2008;32:11591166.

[60] Chaignot C, Zureik M, Rey G, et al. Risk of hospitalisation and death related to baclofen for alcohol use disorders: Comparison with nalmefene, acamprosate, and naltrexone in a cohort study of 165334 patients between 2009 and 2015 in France. Pharmacoepidemiol. Drug Saf. 2018;27:1239-1248.

[61] Reus VI, Fochtmann L, Bukstein O, et al. The American psychiatric association practice guideline for the pharmacological treatment of patients with alcohol use disorder. Am.

Do not disseminate or use without explicit permission from the authors 
Hugo López-Pelayo ${ }^{1}{ }^{*}$, Paola Zuluaga ${ }^{2}$, Elsa Caballeria ${ }^{1}$, Win Van der Brink ${ }^{3}$, Karl Mann $^{4}$, Antoni Gual ${ }^{1 \mathrm{a}}$.

J. Psychiatry. American Psychiatric Association; 2018. p. 86-90.

[62] Sinclair JD. Drugs to decrease alcohol drinking. Ann. Med. 1990;22:357-362.

Do not disseminate or use without explicit permission from the authors 